\title{
Caracterização estrutural e morfológica de compostos químicos para produção vítrea
}

Structural and morphological characterization of chemical compounds for glassy production

\author{
A. L. F. Novais ${ }^{1 *}$; A. P. P. Lima ${ }^{1}$; A. R. S. Santos ${ }^{1}$; R. R. R. Bezerra ${ }^{1}$; F. C. L. \\ Ferreira $^{1}$; D. N. Souza ${ }^{2}$ \\ ${ }^{\text {I}}$ Faculdade de Engenharia Mecânica/Instituto de Geociências e Engenharias/Universidade Federal do Sul e Sudeste, \\ 68505-080, Marabá-Pará, Brasil \\ ${ }^{2}$ Departamento de Física/Universidade Federal de Sergipe, 49100-00, Aracaju-Sergipe, Brasil \\ *andreanovais@unifesspa.edu.br \\ (Recebido em 28 de fevereiro de 2019; aceito em 29 de junho de 2019)
}

\begin{abstract}
No presente trabalho foram realizadas medidas de Difração de Raios X (DRX), Microscopia Eletrônica de Varredura (MEV) e Espectroscopia por Energia Dispersiva (EED) em óxido de alumínio $\left(\mathrm{Al}_{2} \mathrm{O}_{3}\right)$, carbonato de cálcio $\left(\mathrm{CaCO}_{3}\right)$ e nitrato de prata $\left(\mathrm{AgNO}_{3}\right)$. O difratograma do $\mathrm{Al}_{2} \mathrm{O}_{3}$ revelou a fase $\alpha$ ou corindo, a fase observada no $\mathrm{CaCO}_{3}$ foi a calcita, e no $\mathrm{AgNO}_{3}$ foram observadas as fases relacionadas a prata. Todos os difratogramas estudados apresentaram planos cristalinos. A imagem do $\mathrm{Al}_{2} \mathrm{O}_{3}$, obtida por MEV revelou a presença de aglomerados e uma morfologia formada por plaquetas hexagonais, no $\mathrm{CaCO}_{3}$ foi observada uma estrutura com forma romboédrica-hexagonal e no $\mathrm{AgNO}_{3}$ uma morfologia elipsoidal. A análise de EED realizada no $\mathrm{Al}_{2} \mathrm{O}_{3}$ mostrou a presença de alumínio e oxigênio, no $\mathrm{CaCO}_{3}$ foi observado cálcio, carbono e oxigênio. As análises de EED realizadas nas amostras investigadas revelaram compostos livres de impurezas. O presente trabalho tem como objetivo, caracterizar os compostos químicos que serão utilizados na produção de diferentes espécimes vítreas para aplicações dosimétricas.
\end{abstract}

Palavras-chave: técnicas de caracterização, compostos químicos, vidros.

In the present work, measurements of X-ray diffraction (XRD), Scanning Electron Microscopy (SEM) and Energy Dispersive Spectroscopy (EDS) in aluminum oxide $\left(\mathrm{Al}_{2} \mathrm{O}_{3}\right)$, calcium carbonate $\left(\mathrm{CaCO}_{3}\right)$ and silver nitrate $\left(\mathrm{AgNO}_{3}\right)$. The diffractogram of $\mathrm{Al}_{2} \mathrm{O}_{3}$ revealed the $\alpha$ or corundum phase, the phase observed in $\mathrm{CaCO}_{3}$ was calcite, and in $\mathrm{AgNO}_{3}$ the phases related to silver were observed. The $\mathrm{Al}_{2} \mathrm{O}_{3}$ image, obtained by MEV revealed the presence of agglomerates and a morphology formed by hexagonal platelets, a structure with a rhombohedral-hexagonal shape was observed in $\mathrm{CaCO}_{3}$ and in $\mathrm{AgNO}_{3}$ the observed morphology was an ellipsoid. The analysis of EDS carried out in $\mathrm{Al}_{2} \mathrm{O}_{3}$ showed the presence of aluminium and oxygen, in the $\mathrm{CaCO}_{3}$ was observed calcium, carbon and oxygen and in the $\mathrm{AgNO}_{3}$ analysis was not performed. The EDS analyses performed on the investigated samples revealed compounds free of impurities. The present work aims to characterize the chemical compounds that will be used in the production of different vitreous specimens for dosimetric applications.

Keywords: characterization techniques, chemical compounds, glasses.

\section{INTRODUÇÃO}

Segundo Alves et al. (2001) [1] e Novais (2015) [2], os vidros têm sido responsáveis por grandes avanços científicos por apresentarem estrutura amorfa permitindo que sejam adicionados em sua composição concentrações variáveis de diferentes elementos da tabela periódica. A simplicidade de síntese destes materiais, a durabilidade de suas propriedades térmicas, mecânicas e químicas, a capacidade de fabricação de dispositivos óticos de qualquer formato e tamanho, seu custo relativamente baixo, a possibilidade de incorporar impurezas e sua alta homogeneidade ótica os torna ideais para aplicação em dispositivos como, lâmpadas de fósforo, hospedeiros de laser ativos e dosímetros $[3,4,5]$

$\mathrm{A}$ adição de óxido de alumínio $\left(\mathrm{Al}_{2} \mathrm{O}_{3}\right)$ nas estruturas vítreas afeta diferentes propriedades físicas, como temperatura de transição vítrea (Tg), densidade e índice de refração [6]. Venkateswara et al. (2014) [7] mostraram que a inserção do óxido de cálcio $(\mathrm{CaO})$ leva a um aumento significativo da densidade. Mendes et al. (2018) [8] relataram que ao incorporar nitrato de prata $\left(\mathrm{AgNO}_{3}\right) \mathrm{em}$ sistemas vítreos, esses podem ser utilizados como antimicrobactericida. 
Com o avanço da ciência e a profunda compreensão das propriedades físicas e químicas dos vidros acoplados a modernas tecnologias analíticas e de controle, fizeram com que os estudos sobre este material se intensificasse e com isso novos avanços tecnológicos pudessem ser realizados, estes voltados para resolver problemas mais urgentes do mundo, como na área da saúde, energia e águas $[9,10]$.

Deste modo, foram utilizadas técnicas de Microscopia Eletrônica de Varredura (MEV), Espectroscopia por Energia Dispersiva (EED) e Difração de Raios X (DRX) para caracterizar os compostos químicos investigados.

\section{MATERIAL E MÉTODOS}

As análises dos compostos químicos estudados foram realizadas no Laboratório de Caracterização Estrutural do Instituto de Geociências e Engenharias da Universidade Federal do Sul e Sudeste do Pará, IGE/UNIFESSPA. As estruturas cristalográficas dos compostos investigados foram estudadas a partir das análises realizadas em um diafratrômetro de raios $\mathrm{X}$ composto por um sistema Miniflex 600 da marca Rigaku Miniflex 600 (wilth incident \& receiving 2.5 soller slits \& D/tex Ultra), com um gerador de raios X de $600 \mathrm{~W}(20-40 \mathrm{kV}$ e $2-15 \mathrm{~mA}$ variáveis). $\mathrm{O}$ tubo de raios $\mathrm{X}$ utilizado foi de Cobre $(\mathrm{Cu})$ com foco fino de $1 \mathrm{~kW}$ e um goniômetro de tela de $2 \theta$. A fenda utilizada foi soller incidente e recepção de $2.5^{\circ}$, fenda DS 1.25 e $0.625^{\circ}$, fenda DHL $10 \mathrm{~mm}$, fenda SS $1.25^{\circ}$, fenda de $0.3 \mathrm{~mm}$. Foi utilizado um filtro de Ni para K-beta detector e controlador de raio X Beam stopper e fenda de espalhamento de $8 \mathrm{~mm}$. Para o processamento e análise dos dados, foram utilizados os softwares: PDXL Qualitative (busca automática de fichas difratométricas) e PDXL Rietveld acadêmico e um Banco de Dados COD (Crystallography Open Database). Os absorvedores utilizados foram de $\mathrm{Cu}$ e $\mathrm{Al}$ e um detector $\mathrm{D} / \mathrm{teX}$ ultra de alta velocidade e sensibilidade acoplado à fonte de raio $\mathrm{X}$.

Para o estudo da morfologia, foi utilizado um Microscópio Eletrônico de Varredura (MEV) Hitachi Tabletop Microscope (TM-3000) com Espectrômetro de Energia Dispersiva (EDS) swifted3000 acoplado. As variações causadas nas morfologias dos compostos foram investigadas utilizando uma tensão de aceleração de $15 \mathrm{kV}$, tempo de aquisição da imagem de $59,9 \mathrm{~s}$, o tempo de processamento $5 \mathrm{~s}$, fonte de filamento de tungstênio, detector de elétrons retroespalhados com câmara de vácuo e magnificação máxima de 4000x. O EDS utilizado possui detector de silício tipo sdd de $30 \mathrm{~mm}^{2}$ e resolução $161 \mathrm{ev}$ (cu-k $\alpha$ ) com analisador multicanal 2048 canais (10 ev/canal). Todas as amostras foram dispostas em disco de alumínio (stub), sem fita condutora em baixo vácuo. Os componentes principais analisados foram: $\mathrm{Al}, \mathrm{O}, \mathrm{Ca}, \mathrm{C}, \mathrm{Ag}, \mathrm{N}$, cujos resultados foram gerados em imagens de pontos (dot mapping) e histogramas quantitativos (sum spectrum).

Os compostos químicos analisados foram: óxido de alumínio $\left(\mathrm{Al}_{2} \mathrm{O}_{3}\right)$, carbonato de cálcio $\left(\mathrm{CaCO}_{3}\right)$ e o nitrato de prata $\left(\mathrm{AgNO}_{3}\right)$, todos os reagentes foram adquiridos na Sigma Aldrich com $99,9 \%$ de pureza. Para a realização das análises, todos os compostos foram passados em uma peneira com granulometria de $175 \mu \mathrm{m}$, para minimizar orientação preferencial dos planos cristalinos e garantir a sua homogeneidade, antes da realização da análise.

\section{RESULTADOS E DISCUSSÃO}

Os resultados obtidos por DRX nas amostras de $\mathrm{Al}_{2} \mathrm{O}_{3}, \mathrm{CaCO}_{3}$ e $\mathrm{AgNO}_{3}$ tem por finalidade identificar e estudar as fases cristalinas dos citados compostos. Os difratogramas do $\mathrm{Al}_{2} \mathrm{O}_{3}, \mathrm{CaCO}_{3}$ e $\mathrm{AgNO}_{3}$ foram obtidos na faixa de (20) $10^{\circ}$ e $90^{\circ}$ e apresentaram picos fortes característicos de suas estruturas cristalinas e ausência de fases amorfas. A Figura 1 mostra o padrão de difração de raios $\mathrm{X}$ do óxido de alumínio $\left(\mathrm{Al}_{2} \mathrm{O}_{3}\right)$ e os picos em $2 \theta$ com seus respectivos planos cristalinos $25,670^{\circ}[012], 35,424^{\circ}[104], 37,813^{\circ}$ [110], 41,482 ${ }^{\circ}[006], 43,615^{\circ}$ [113], 46,317 ${ }^{\circ}$ [202], 52, $118^{\circ}$

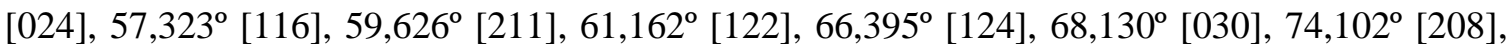
$76,605^{\circ}[119]$. 


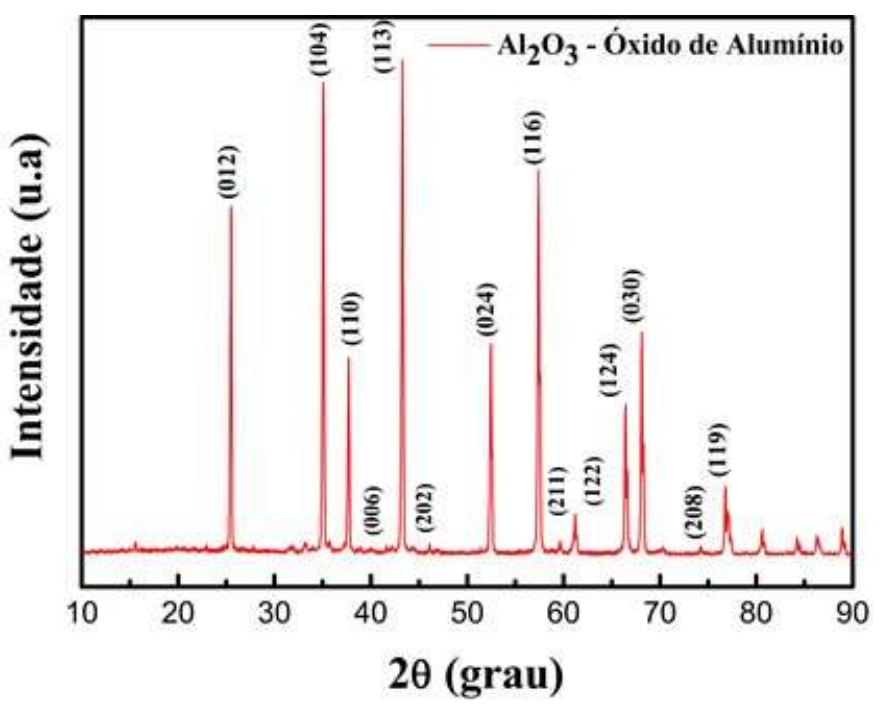

Figura 1. Difratograma do $\mathrm{Al}_{2} \mathrm{O}_{3}$.

A Figura 1 revela a fase $\alpha-\mathrm{Al}_{2} \mathrm{O}_{3}$, ou corindo, que é a fase mais estável da alumina [11]. O corindo tem uma estrutura romboédrica na qual os íons de oxigênio formam uma sub-rede hexagonal compacta com os íons de alumínio ocupando $2 / 3$ dos sítios intersticiais octaédricos [12].

A Figura 2 mostra o padrão de difração de raios $\mathrm{X}$ do carbonato de cálcio $\left(\mathrm{CaCO}_{3}\right)$ que é um biomaterial inorgânico [13]. O carbonato de cálcio pode formar polimorfos cristalinos estáveis à temperaturas e pressões ambientes (calcita, aragonita) e instável termodinamicamente (veterita) $[13,14,15]$. A Figura 2 mostra ainda, os picos em $2 \theta$ com seus respectivos planos cristalinos

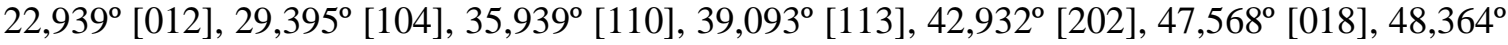
[116], 57,465 ${ }^{\circ}$ [122], 60,678 ${ }^{\circ}[214], 64,319^{\circ}$ [300].

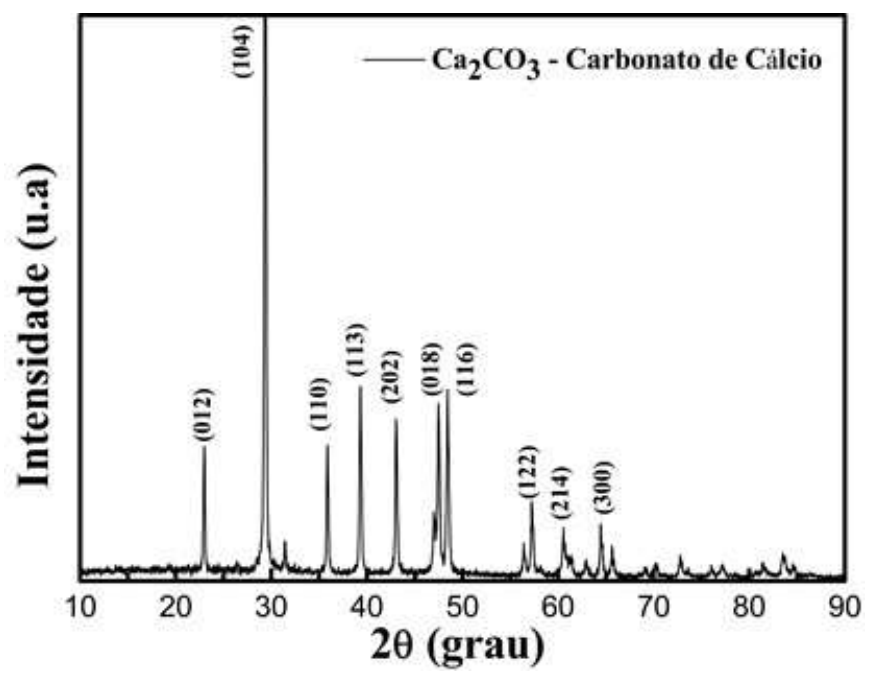

Figura 2. Difratograma do $\mathrm{CaCO}_{3}$.

A partir da Figura 2 é possível observar a fase mais estável termodinamicamente do carbonato de cálcio que é a calcita [13, 16, 17], essa fase é confirmada pelo valor de $2 \theta$ em $29,395^{\circ}$ onde mostra que a calcita está bem caracterizada na forma de $\mathrm{CaCO}_{3}$ [18]. A pureza do carbonato de cálcio analisado neste trabalho foi confirmada a partir da comparação com o espectro de DRX do Sitepu [19].

A Figura 3 mostra o padrão de difração de raios $\mathrm{X}$ do nitrato de prata $\left(\mathrm{AgNO}_{3}\right)$ e os picos em $2 \theta$ com seus respectivos planos cristalinos $39,178^{\circ}$ [111], 43,615 ${ }^{\circ}$ [200], 62,328 ${ }^{\circ}$ [220], 80,472 ${ }^{\circ}$ [311]. Os picos encontrados possuem uma relação com a fase $\mathrm{Ag}$ presente no $\mathrm{AgNO}_{3}$. 




Figura 3: Difratograma do $\mathrm{AgNO}_{3}$.

A Figura 4a mostra a imagem obtida por MEV da estrutura do óxido de alumínio $\left(\mathrm{Al}_{2} \mathrm{O}_{3}\right)$, também conhecido como alumina. Na Figura $4 \mathrm{~b}$ pode ser observado o espectro de energia da alumina obtido por EED.

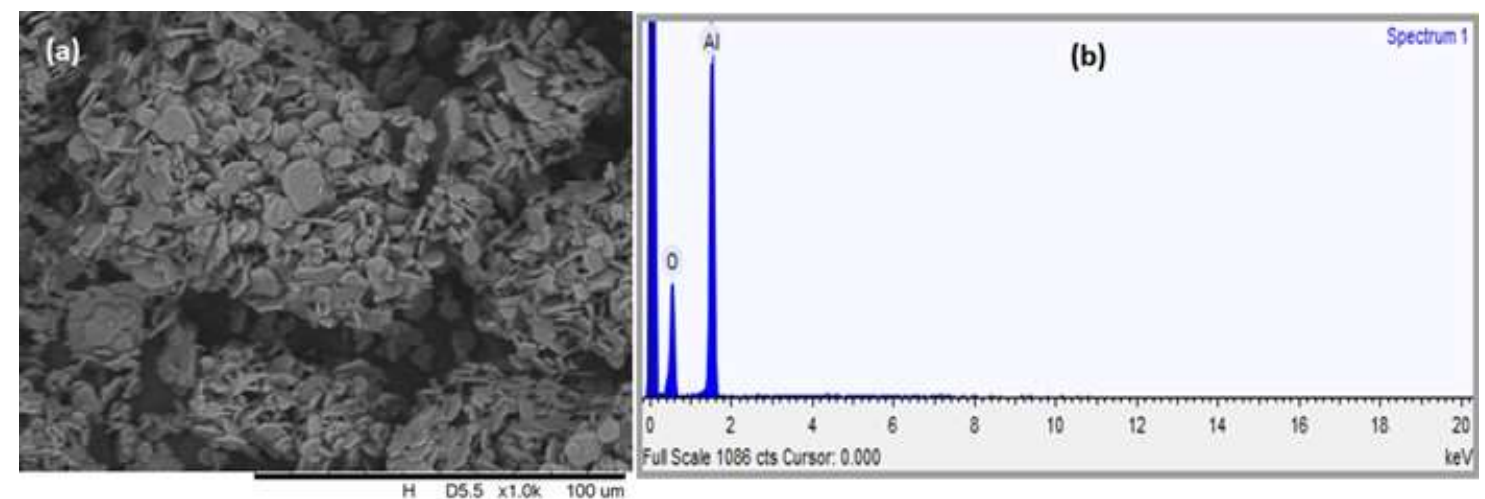

Figura 4. (a) Imagem do $\mathrm{MEV}$ do $\mathrm{Al}_{2} \mathrm{O}_{3}$ e (b) Análise de $\mathrm{EED}$ do $\mathrm{Al}_{2} \mathrm{O}_{3}$.

A imagem da amostra de $\mathrm{Al}_{2} \mathrm{O}_{3}$ observada na Figura 4a indica a presença de aglomerados e uma morfologia formada por plaquetas hexagonais [11]. A Figura $4 \mathrm{~b}$ mostra os resultados da análise EED para alumina sem tratamento eletro-térmico. Como pode ser observado nos picos de energia nesta figura, os elementos encontrados na amostra de alumina correspondem apenas ao alumínio e ao oxigênio o que podemos inferir que a amostra investigada não apresenta impurezas.

A Figura 5a mostra a imagem obtida por MEV da estrutura do carbonato de cálcio $\left(\mathrm{CaCO}_{3}\right)$. $\mathrm{Na}$ Figura 5 b pode ser observado o espectro de energia do $\left(\mathrm{CaCO}_{3}\right)$ obtido por EED.

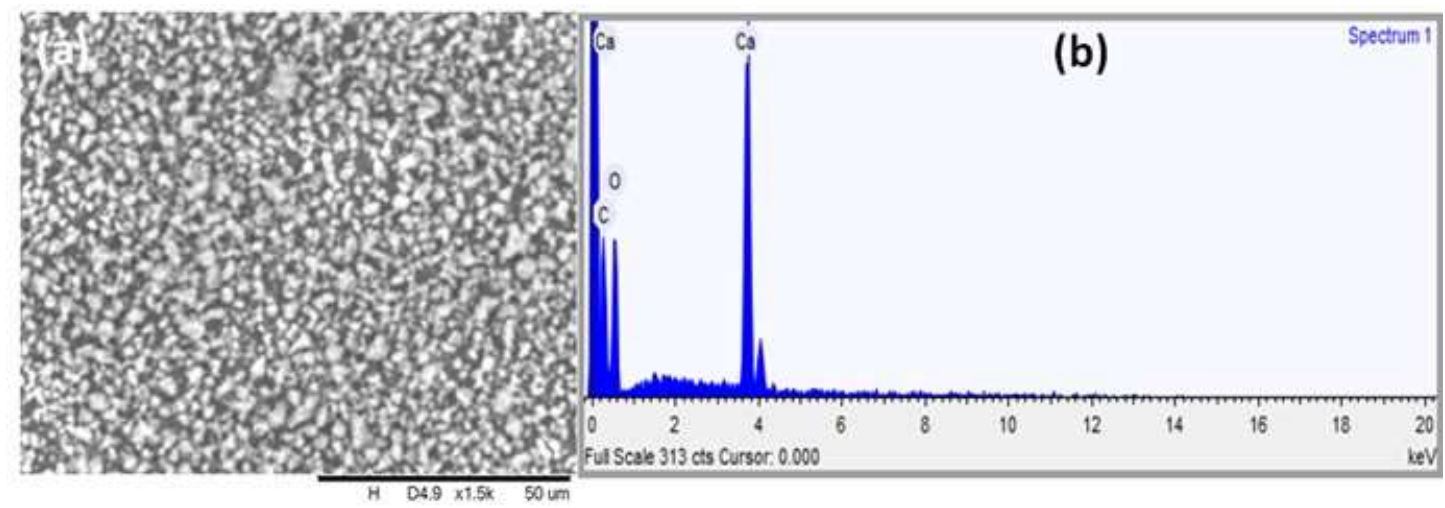

Figura 5 (a) Imagem de $\mathrm{MEV}$ do $\mathrm{CaCO}_{3}$ e (b) Análise de $\mathrm{EED}$ do $\mathrm{CaCO}_{3}$ 
A imagem da amostra de $\mathrm{CaCO}_{3}$ observada na Figura 5a indica uma estrutura com morfologia romboédrica-hexagonal, em que o comprimento e o diâmetro dos cristais são aproximadamente iguais e os cristais podem estar agregados ou não [20,21, 22]. A Figura 5 b mostra os resultados da análise de EED para o $\mathrm{CaCO}_{3}$. Como pode ser observado nos picos de energia nesta figura, o cálcio, o carbono e o oxigênio constituem os únicos elementos químicos presentes no composto, o que nos leva a inferir que a amostra de $\mathrm{CaCO}_{3}$ analisada não apresenta impurezas. [23].

A Figura 6 mostra a imagem obtida por MEV da estrutura do nitrato de prata $\left(\mathrm{AgNO}_{3}\right)$.

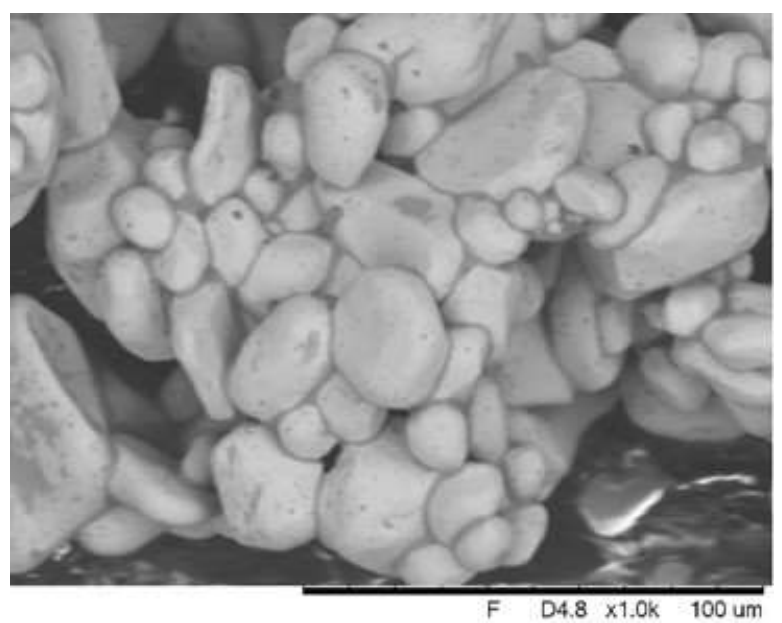

Figura 6. Imagem de $\mathrm{MEV}$ do $\mathrm{AgNO}_{3}$.

A imagem da amostra de $\mathrm{AgNO}_{3}$ observada na Figura 6 mostra uma morfologia elipsoidal, essa é uma das diversas formas em que as nanopartículas de prata podem ser obtidas a partir do $\mathrm{AgNO}_{3}$. Além da forma elipsoidal, as nanopartículas também podem ser produzidas nas seguintes formas: esférica, bastão, estrela, triângulo e tubo [24].

\section{CONCLUSÃO}

Os resultados obtidos a partir dos difratogramas de raios X revelaram: a fase mais estável $(\alpha)$ do $\mathrm{Al}_{2} \mathrm{O}_{3}$, a fase mais estável termodinamicamente do $\mathrm{CaCO}_{3}$, que é a calcita e a fase $\mathrm{Ag}$ presente no $\mathrm{AgNO}_{3}$. Todos os difratogramas estudados apresentaram picos fortes e ausência de fases amorfas, confirmando, a natureza cristalina dos compostos investigados.

As observações realizadas nas imagens obtidas por $\mathrm{MEV}$ do $\mathrm{Al}_{2} \mathrm{O}_{3}$ mostraram aglomerados e uma morfologia formada por plaquetas hexagonais, na imagem de $\mathrm{CaCO}_{3}$ foi observada uma morfologia romboédrica-hexagonal e na imagem do $\mathrm{AgNO}_{3}$ uma morfologia elipsoidal.

As análises dos elementos realizadas por EED revelaram que o espectro de energia do $\mathrm{Al}_{2} \mathrm{O}_{3}$ era composto apenas por alumínio e oxigênio, no $\mathrm{CaCO}_{3}$ pelo cálcio, carbono e oxigênio, não foi realizada análise de EED no $\mathrm{AgNO}_{3}$. A partir da EED foi possível inferir que os compostos investigados estavam livres de impurezas.

Por fim, os resultados mostraram a importância de conhecer previamente a estrutura e a morfologia dos compostos químicos que serão utilizados na produção de espécimes vítreas e assim, auxiliar no controle de propriedade físicas como, pressão, temperatura e volume.

\section{AGRADECIMENTOS}

Os autores agradecem a Coordenação de Aperfeiçoamento de Pessoal de Nível Superior (CAPES), ao Laboratório de Caracterização (LC) da Universidade Federal do Sul e Sudeste do Pará (UNIFESSPA), ao Laboratório de Física Médica (LFM) da Universidade Federal de Sergipe (UFS). 


\section{REFERÊNCIAS BIBLIOGRÁFICAS}

1. Alves OL, Gimenez IF, Mazali IO. Vidros. Cadernos Temáticos de Química Nova na Escola. 2001 fev; 09-20.

2. Novais ALF, Dantas NO, Guedes I, Vermelho MVD. Spectroscopic properties of highly Nd-doped lead phosphate glass. J Alloys Compounds 2015;648:338-345. doi:10.1016/j.jallcom.2015.06.148.

3. Jafari M, Bradley DA, Gouldstone A, Sharpe PHG, Alalawi A, Jordan TJ, Clark CH, Nisbet A, Spyrou NM. Low-cost commercial glass beads as dosimeters in radiotherapy. Rad Phys Chem. 2014;97:95-101. doi:10.1016/j.radphyschem.2013.11.007

4. Sontakke AD, Ueda J, Tanabe $\mathrm{S}$. Effect of synthesis conditions on $\mathrm{Ce}^{3+}$ luminescence in borate glasses. $\mathrm{J}$ Non-Crystal Sol. 2015;431:150-153. doi:10.1016/j.jnoncrysol.2015.04.005

5. Tripathi H, Kumar AS, Singh SP. Preparation and characterization of $\mathrm{Li}_{2} \mathrm{O}-\mathrm{CaO}-\mathrm{Al}_{2} \mathrm{O}_{3}-\mathrm{P}_{2} \mathrm{O}_{5}-\mathrm{SiO}_{2}$ glasses as bioactive material. Bull Mater Sci. 2016;39(2):365-376. doi:10.1007/s12034-016-1154-6

6. Yue Y, Wang Y, Cao Y, Chen S, Zhou Q, Chen W, Hu L. Effect of $\mathrm{Al}_{2} \mathrm{O}_{3}$ on structure and properties of $\mathrm{Al}_{2} \mathrm{O}_{3}-\mathrm{K}_{2} \mathrm{O}-\mathrm{P}_{2} \mathrm{O}_{5}$ glasses. Opt Mat Exp. 2018;8(2):245-258. doi:10.1364/OME.8.000245

7. Venkateswara GR, Shashikal HD. Optical and mechanical properties of calcium phosphate glasses. Glass Physics and Chemistry. 2014; 40(3):303-309. doi: 10.1134/S1087659614030249

8. Mendes E, Mendes E, Savi1 GD, Angioletto GD, Riella HG, Fiori MA. High performance bactericidal glass: evaluation of the particle size and silver nitrate concentration effect in ionic exchange process. Cerâmica. 2018;64(370):156-165. :doi:10.1590/0366-69132018643702197

9. Morse DL, Evenson JW. Welcome to the Glass Age. Int J Applied Glass Science. 2016;7(4):409-412. doi:10.1111/ijag.12242

10. Pitaluga LH, Souza MT, Zanotto ED, Romero MS, Hatton P. Electrospun F18 Bioactive Glass/PCL Poly ( $\varepsilon$-caprolactone) - Membrane for Guided Tissue Regeneration. Materials. 2018;11(3):E400. doi: $10.3390 / \mathrm{ma} 11030400$

11. Souza PS, Santos HS, Toledo SP. Standard Transition Aluminas. Electron Microscopy Studies. Standard Transition Aluminas. Electron Microscopy Studies. Materials Research, 2000;4(3):104-114 doi.org/10.1590/S1516-14392000000400003

12. Boumaza A, Favaro L, Lédion J, Sattonnay G, Brubach JB, Berthet P, Huntz AM, Roy P, Tétot R. Transition alumina phases induced by heat treatment of boehmite: An X-ray diffraction and infrared spectroscopy study. Journal of Solid State Chemistry 2009; (182) :1171-1176. doi:10.1016/j.jssc.2009.02.006

13. Hariharan Manjusha, Varghese N, Cherian DAB, Sreenivasan DPV, Jenish P, Asmy Antony. K.A. Synthesis and Characterisation of $\mathrm{CaCO}_{3}$ (Calcite) Nano Particles from Cockle Shells Using Chitosan as Precursor. International Journal of Scientific and Research Publications, 2014 1(4) ISSN 2250-3153 www.ijsrp.org

14. Estroff LA, Leiserowitz L, Addadi L, Weiner S, Hamilton AD. Characterization of an organic hydrogel: a cryo-TEM and X-ray diffraction study. Org. Biomol. Chem, 2003;2:137-141.

15. Gauldie, RW, Sharma SK., Volk E, Micro-Raman spectral study of vaterite and aragonite otoliths of the coho salmon, Oncorhynchus kisutch. Comp. Biochem. Physiol. A Comp. Physiol. 1997;(118):753-757.

16. Islam KN, Zuki ABZ, M. M. Noordin, M. Z. B. Hussein, N. S. S. B. A. Rahman, and M. E. Ali, "Characterisation of calcium carbonate and its polymorphs from cockle shells (Anadara granosa)," Power Technology, 2011;1-3 (213):188-191.

17. Islam KhN, Zuki ABZ, Ali ME, Hussein MZB, Noordin. Loqman MMY, Wahid HW, Hakim MAH, and Hamid SBA. Facile Synthesis of Calcium Carbonate Nano particles from Cockle Shells, Journal of Nano materials 2012. doi.org/10.1155/2012/534010

18. Kilic S. Synthesis of Stable Nano Calcite Journal of the Turkish Chemical Society Section A: Chemistry JOTCSA. 2018;5(2): 869-880. doi.org/10.18596/jotcsa.371374

19. Sitepu H, BH. O'Connor, DLi, Comparative evaluation of the March and generalized spherical harmonic preferred orientation models using X-ray diffraction data for molybdite and calcite powders, J. Appl. Crystallogr, 2005;38:158e167. doi:10.1107/S0021889804031231

20. Marthur VK. High speed manufacturing process for precipitated calcium carbonate employing sequential pressure carbonation., US patente, n. 6251356, 2001.

21.Duport LG, Briones MJI, Briones Rodríguez, Covelo JB. Amorphous calcium carbonate biomineralization in the earthworm's calciferous gland: Pathways to the formation of crystalline phases. Journal of Structural Biology 162 (2008) 422-435. doi:10.1016/j.jsb.2008.02.007

22. Borowitzka MA, Larkum AWD and Nockolds CE. A scanning electron microscope study of the structure and organization of the calcium carbonate deposits of algae. Phycologia, 1974;13 (3):195-203. doi.org/10.2216/i0031-8884-13-3-195.1 
23. Sá MD, Santos FSF. Ferreira, V. P. Fook MVL. Análise química, térmica e morfológica de compostos obtidos com PEEK/CaCO3. Revista Eletrônica de Materiais e Processos, 2016;1(11) 49-53 ISSN 18098797

24. Rao GVSN, Mantravadia KM and Oztekinb Y. Strategies to synthesize various nanostructures of silver and their applications - a review Umme Thahira Khatoon. Royal Society of Chemistry, 2018;(8):197391975. doi: 10.1039/c8ra00440d 